\title{
APPLIANCES
}

\section{NEW DESIGN IN ANTERIOR CHAMBER LENSES*}

BY

\author{
T. G. WYNNE PARRY \\ Bangor, North Wales
}

MORE and more attention is being paid to the question of intra-ocular acrylic implants since Ridley's original venture into this field.

More recently, interest has changed from Ridley's original idea of an acrylic lens replacing the extracted lens in position behind the pupil to the anterior chamber implant.

It is possible that this has happened because of the greater ease of insertion of the anterior chamber lenticulus and the fact that it is easier to take such a lens out should anything go wrong; furthermore, the anterior chamber lens has the great advantage over Ridley's lens of being capable of insertion after an intra-capsular operation or a cataract extraction with full iridectomy or both.

The best known of the anterior chamber lenses in use at present is the Strampelli lens, which is made entirely in plastic; the shape being approximately as shown in Fig. 1.

Fig. 1.-Shape of lens.

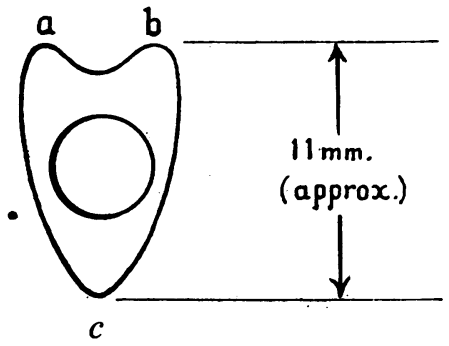

It is inserted into the anterior chamber through a small incision and is held in position by each of the three projecting points $(a, b, c)$ being jammed into the drainage angle between the root of the iris and the cornea. There are other modifications of this lens, some with three legs and some with spring loops of various materials, but essentially the mode of fixation is the same, by means of pressure against the iris root and cornea in the region of the filtration angle.

Owing to the exact fit required in this type of lens, it is necessary to make two or three lenses for each case and, by trial and error, to select the one that fits not too loosely and not too tightly, with just the right pressure in the angle.

* Received for publication July $15,1957$. 
I have felt for the last year or more that a lens which relies for its fixation on pressure in such a delicate part of the eye as the root of the iris cannot be entirely free from the danger of causing iris atrophy and possibly other troubles.

The new type of lens described below has therefore been designed with the following objects:

(1) To avoid the dangers of pressure in the filtration angle.

(2) To be easy to insert and, if necessary, to extract.

(3) To be capable of implantation at the time of the extraction of the cataract if desired; this is hardly possible with the Strampelli lens.

(4) To avoid the necessity for making a series of lenses of different sizes for each case to ensure a good fit.

Construction.-The lens (Fig. 2, $a$ and $b$ ), made of Transpex I (I.C.I.), is held in a small tantalum frame with two projecting arms; the end of each arm has a small downward projection, similar to a letter " $L$ " lying on its side; these are also bent backwards at an angle of approximately $145^{\circ}$.

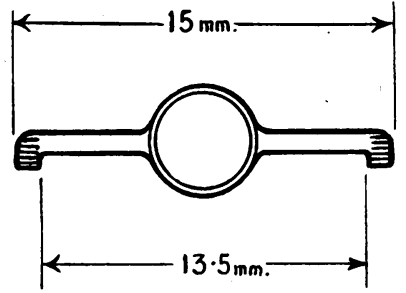

(a)

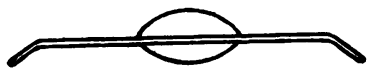

(b)

Fig. 2.-Lens in tantalum frame. (a) Front view. (b) Side view with front upwards.

The width of the frame from the end of one arm to the end of the other is $15.5 \mathrm{~mm}$., and the distance between the downward projections is $13 \mathrm{~mm}$. The vertical width of the arms is $1.5 \mathrm{~mm}$. A line drawn along the lower edges of the arms passes through the centre of the lens.

Technique of Insertion.-A small conjunctival flap is raised on both sides of the cornea and dissected well on to the cornea, as for a trephine operation. At the side, where it is intended to insert the lens into the anterior chamber, a corneo-scleral mattress suture is placed. Originally, a Beers's knife was used for the section of the cornea; the knife was passed through the corneo-sclera as close to the iris as possible without touching it, and the point emerged on the opposite side of the anterior chamber at a corresponding position, being passed through far enough to make the wound on the opposite side large enough to allow the passage of one of the tantalum arms of the frame.

The level of the lower part of the section of the Beers's knife passed in front of the centre of the pupil, so that the centre of the acrylic lens was on the same plane. 
A Beers's knife was used so that there could be a large enough incision on the "inserting side" for the lens itself to pass through, whereas only a small incision was necessary on the other side for the leading tantalum arm to pass into.

The arm of the lens is seized at the tip with a pair of forceps made for the purpose and, with the large incision held open, the lens is passed across the anterior chamber and the end of the leading tantalum arm is engaged in the incision on the opposite side. The lens is then moved downwards until the lower edges of the arms rest at the bottom of the two incisions and the lens is centred in front of the pupil. The small downward projections at the end of each arm prevent the lens sliding out to one side or the other.

The corneo-scleral mattress suture is drawn tight and the bent ends of the arms are adjusted to the curve of the sclera if the angle at which they are bent is too great. This is done by slipping the point of a von Graefe or other knife under the little projecting tips and gently pressing the angle out with any small blunt instrument.

The conjunctival flaps are now sutured back into place and the anterior chamber may be refilled with a penicillin solution. Atropine can be safely used and the usual toilet of the eye performed afterwards. Figs 3 and 4 (opposite) show the lens in situ.

It has been found better in a recent case to use a straight-backed Smith's von Graefe knife for the first section, as this gives a cleaner flatter wound, and it also makes it possible to keep the incisions strictly parallel to the face of the iris; this is important as the two flat tantalum arms will be sealed in this position later.

After the incision with the von Graefe knife, the wound on the side at which it is proposed to introduce the lens can be enlarged with corneo-scleral scissors.

Healing.-For some years I used tantalum drains, which were made in the form of a channel and were inserted under the conjunctiva through the corneo-sclera into the anterior chamber. In spite of their shape, which should have facilitated drainage, the wound effectively sealed up these drains after some months. I do not, therefore, anticipate that there will be the least leakage of aqueous through the wounds in which the thin and flat tantalum arms are sealed in place.

Tolerance.-It is now recognized that tantalum is tolerated extremely well in the anterior chamber, and I have noticed no change in tantalum drains which have been left in the eye over several years. The tolerance of the eye to the material of the lens has, of course, had a fairly extensive trial with both the Ridley and Strampelli lenses. There should, therefore, be no fear of reaction to either of these materials in the eye.

Refraction.-If the lens is inserted at the time of the extraction, then a lens equivalent to approximately $+10 \mathrm{D}$ can be put in, but, if it is desired 
Fig. 3.-Diagram of lens in situ. •
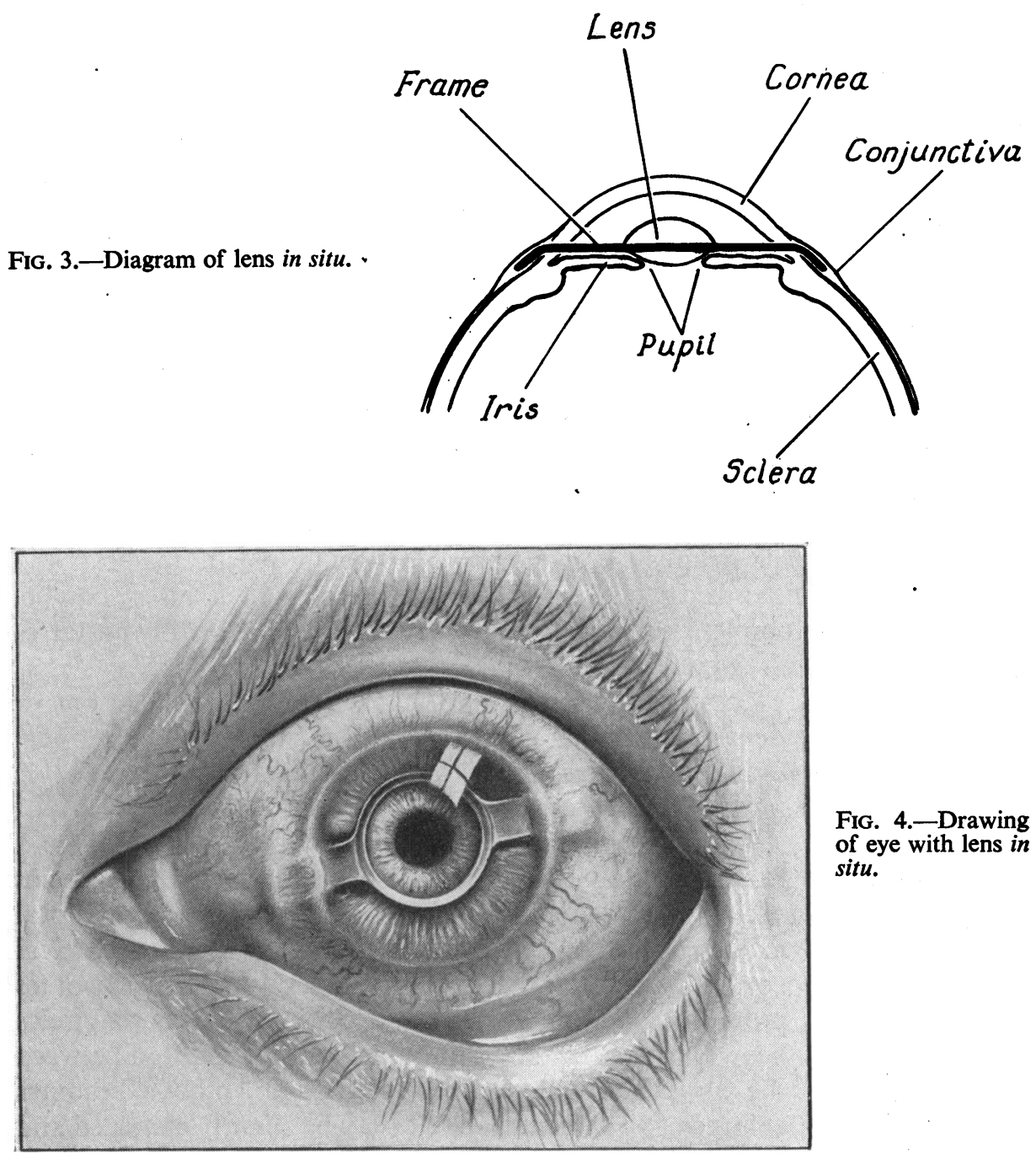

Fig. 4.-Drawing of eye with lens in situ.

to put the lens in at a later date, the lens can be ground to the patient's refraction.

Asepsis.-I have found it satisfactory first to clean the lens gently with a soft camel hair brush with warm soap and water and then to rinse it thoroughly with sterile water, place it in a solution of cetrimide for 10 to 15 minutes, rinse it again with sterile water, and finally place it in a concentrated solution of penicillin for half an hour or more.

The lenses are manufactured by Messrs. G. Nissel \& Company, Siddons Lane, Baker Street, London, N.W., and the special forceps by Messrs. Dixey, 19, Wigmore Street, London, W.1. I owe my thanks to both firms for the time and trouble they have taken. 